\title{
Ectopic expression of the Streptomyces coelicolor whiE genes for polyketide spore pigment synthesis and their interaction with the act genes for actinorhodin biosynthesis
}

\author{
Tin-Wein Yut and David A. Hopwood
}

John Innes Centre, Norwich Research Park, Colney, Norwich NR4 7UH, UK
Author for correspondence: David A. Hopwood.Tel: +44 1603 452571. Fax: +44 1603456844.

The whiE gene cluster of Streptomyces coelicolor is normally expressed shortly before sporulation in the aerial mycelium, leading to production of the grey polyketide spore pigment. By placing the whiE genes under the control of the thiostrepton-inducible tipA promoter, they were artificially expressed on plasmids or in the chromosome during vegetative growth in a strain deleted for the act genes, which control biosynthesis of the polyketide antibiotic actinorhodin. Certain combinations of whiE-ORFI-VII led to production of mycelial pigments; these were exported into the medium when whiE-ORFI was absent, but poorly in its presence. Combined with comparative sequence data, the results allowed deductions to be made, or confirmed, about the normal roles of the eight known genes, whiE-ORFI-VIII, as follows: whiE-ORFIII, IV, $V$ encode the three components (ketosynthase, chain length factor and acyl carrier protein) of the whiE ' minimal' polyketide synthase (PKS) needed for assembly of the carbon chain of the spore pigment precursor; whiE-ORFII, VI, VII are likely to be involved in cyclizations of the nascent carbon chain; whiEORFVIII controls a late step in the spore pigment biosynthetic pathway, probably a hydroxylation; and whiE-ORFI may encode a protein needed for correct targeting or retention of spore pigment at an appropriate cellular location. In other experiments, genes encoding components of the act-PKS and whiE-PKS were artificially co-expressed. Each of the three whiE minimal PKS subunit genes could complement lesions in the corresponding act-PKS genes to produce actinorhodin or related mycelial pigments, and each of the three act minimal PKS genes could complement lesions in the whiE minimal PKS genes to cause spore pigmentation. Thus the two sets of PKS subunits, which are encoded by genes that have presumably diverged from a common ancestor, are still capable of biochemical 'cross-talk', but this is normally prevented because the gene sets are expressed in different 'tissues' of the differentiated Streptomyces colony. Ectopic expression of sets of whiE-PKS genes presumed to be sufficient to assemble a carbon chain caused inhibition of early growth of the strains, perhaps by causing interference with fatty acid biosynthesis; this yielded circumstantial evidence that the whiE-PKS gene products can also interact with those of the fatty acid synthase(s) of the organism.

Keywords: antibiotic biosynthesis, polyketides, spore pigment, Streptomyces coelicolor, whiE genes 


\section{INTRODUCTION}

Spore colour in Streptomyces coelicolor A3(2) (Davis \& Chater, 1990) and Streptomyces balstedii (Blanco et al., 1992, 1993) apparently depends on the presence of one or more polycyclic aromatic polyketides. Attempts to purify the spore pigments have failed (Brian, 1992), perhaps because they are covalently bound to macromolecular spore components. Deductions about the chemical nature of the pigments rest instead on the discovery that mutations abolishing or modifying spore pigmentation reside in a cluster of genes in each species - named $w b i E$ and $s c b$ respectively - that resemble, in arrangement and sequence, the sets of genes encoding the subunits of the so-called type II polyketide synthases (PKSs) for Streptomyces aromatic antibiotics, including those for actinorhodin in S. coelicolor itself (Fig. 1). These PKS genes resemble in turn genes for fatty acid synthases (FASs). Indeed, there is now ample evidence not only that the biochemistry of fatty acid and polyketide synthesis, in which carbon chains are formed by the successive condensation of small acyl building units, are mechanistically related (O'Hagan, 1991), but that the genes have a common evolutionary origin (Hopwood \& Sherman, 1990; Hopwood \& Khosla, 1992; Katz \& Donadio, 1993; Smith, 1994). Amongst the actinorhodinlike PKSs, the basis of PKS 'programming' is currently being elucidated (McDaniel et al., 1993a, 1994; Shen \& Hutchinson, 1993). Programming refers to the mechanism by which each different PKS controls the appropriate carbon chain length, degree and regiospecificity of ketogroup reduction, and cyclization pattern of its product. It turns out that three subunits of the enzyme, which constitute the 'minimal' PKS and are the ketosynthase (KS), chain length factor (CLF) and acyl carrier protein (ACP), are responsible for building the carbon chain and largely for establishing its first critical cyclization. These subunits are encoded by actI-ORFI, -ORF2 and -ORF3, respectively, in the actinorhodin cluster and by their homologues in the other gene clusters (Fig. 1). Additional subunits of the PKS are responsible for further cyclizations of the nascent carbon chain (McDaniel et al., 1994). Some, but not all, PKSs also include a ketoreductase (KR), which reduces a specific keto-group.

The known extent of the whiE cluster of $S$. coelicolor consists of an operon of seven genes (wbiE-ORFI-VII) and at least one divergently transcribed gene, whiEORFVIII (Davis \& Chater, 1990; Fig. 1). Normally the whiE genes are transcribed just before sporulation in the aerial mycelium (G. H. Kelemen, personal communication). In order to express the genes at other times, and so to gain insight into their roles in spore pigment biosynthesis, we inserted the thiostrepton-inducible tip $A$ promoter (Murakami et al., 1989) into the whiE cluster either on plasmids or in the chromosome. By adding thiostrepton to the medium we could then observe production of $w b i E$-derived pigments during vegetative growth. Study of mutants in which various of the whiE genes had been deleted allowed deductions to be made about the roles of individual genes in pigmentation. This was conveniently done in a strain from which the entire
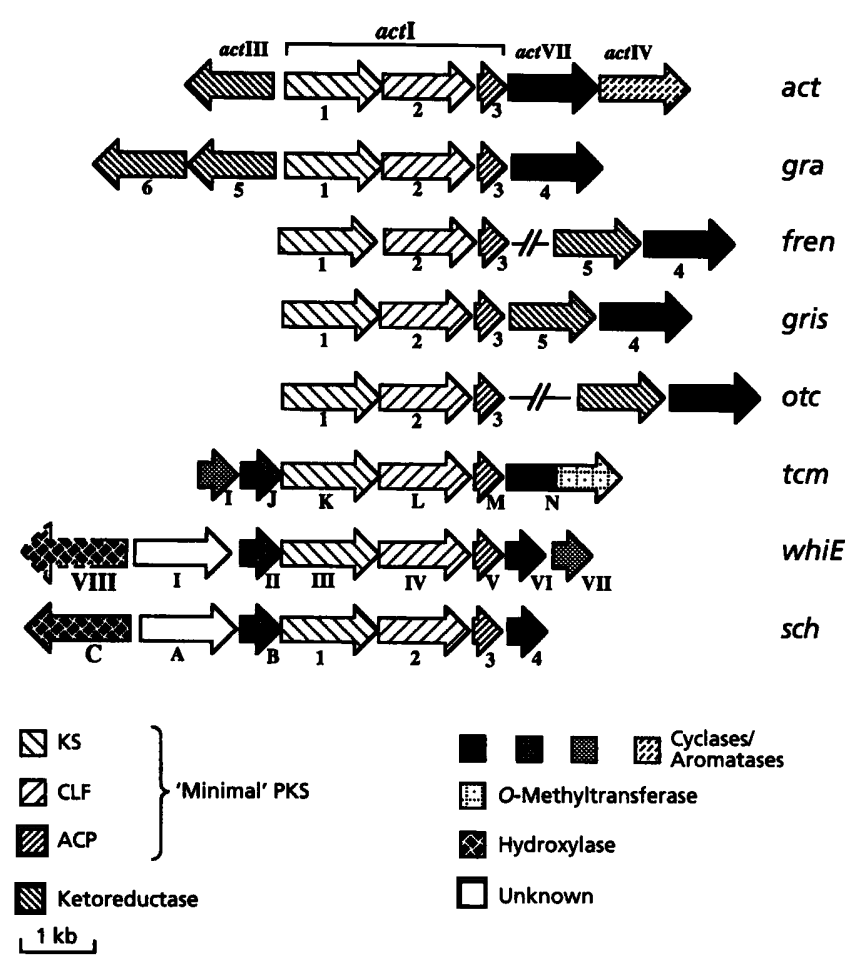

Fig. 1. Organization of genes for aromatic PKS components from Streptomyces spp.: actinorhodin (act) from $S$. coelicolor (Fernández-Moreno et al., 1992), granaticin (gra) from $S$. violaceoruber (Sherman et al., 1989), frenolicin (fren) from $S$. roseofulvus (Bibb et al., 1994), griseusin (gris) from S. griseus (Yu et al., 1994), oxytetracycline (otc) from $S$. rimosus (Kim et al., 1994a), tetracenomycin (tcm) from $S$. glaucescens (Bibb et al., 1989), the whiE-encoded spore pigment from $S$. coelicolor (Davis \& Chater, 1990; Blanco et al., 1993) and the sch-encoded spore pigment from S. halstedii (Blanco et al., 1992, 1993). The arrows depict the relative sizes of the genes and are shaded to represent their functions. The symbol//indicates that one or more genes lie between those shown.

set of act genes had been deleted so that the effects of expressing the whiE genes could be observed in the absence of possible interactions with the products of genes encoding the act-PKS.

Other experiments were designed to study the functional relationships between the act- and whiE-PKS genes, capitalizing on the presence, in the same streptomycete host, of the two sets of related genes that are expressed at different stages in the life-cycle of the organism to produce antibiotic and spore pigments respectively. Building on earlier findings that 'hybrid' PKSs can arise by complementation between subunits of synthases from two different streptomycetes producing structurally related aromatic polyketide antibiotics (Sherman et al., 1992; Khosla et al., 1993), genes for subunits of the act- and $w h i E-P K S s$ were artificially co-expressed. It was found that each of the three subunits of the whiE minimal PKS could complement lesions in the act-PKS to produce actinorhodin (and perhaps related compounds). Conversely, the corresponding act-PKS subunits could complement mutations in the whiE locus to restore spore pigmentation. These results, building on those of Kim $e t$ 
al. (1994b), introduce a favourable system for the further investigation of differential gene expression during the development of a complex prokaryote (Chater, 1993).

\section{METHODS}

Bacterial strains and plasmids. The Escherichia coli hosts (Sambrook et al., 1989) were DH5 $\alpha$ for the manipulation of plasmid DNA and JM101 for the preparation of phagemid DNA. The non-methylating (dam dcm bsdM) strain ET12567 (MacNeil et al., 1992) was used to obtain DNA for transformation of $S$. coelicolor. $S$. coelicolor strains are described in Table 1, and plasmids in Table 2.

Propagation of organisms and general genetic and recombinant DNA techniques. Handling of E. coli strains and general recombinant DNA methods were as described by Sambrook $e t$ al. (1989). For Streptomyces the methods and media described by Hopwood et al. (1985) were used. For studying the pigmentation of the colonies or medium, R2 agar was found to be the most suitable. For analysis of spore colour, cultures were grown either on minimal medium (MM) with mannitol replacing glucose as carbon source and supplemented with 'tiger milk', or on SFM (per litre: $20 \mathrm{~g}$ mannitol, $20 \mathrm{~g}$ soya flour, $20 \mathrm{~g}$ agar), both of which encourage sporulation. Where relevant, thiostrepton was added at $5 \mu \mathrm{g} \mathrm{m}{ }^{-1}$ to induce the tip $A$ promoter(s).

Construction of the ptipA divergent promoter cassette. A fragment carrying $\mathrm{p} t i p A$, with the $\lambda \mathrm{t}_{0}$ transcriptional terminator upstream of it, was excised as an $S_{s t} \mathrm{II}$ (blunt-ended)-EcoRI fragment from pIJ6017 and ligated into pAK113, which also carries ptip $A$ (Murakami et al., 1989), cut with $S m a \mathrm{I}$ and $E c o$ RI to yield pIJ4267 (Fig. 2). The resulting ptip $A$ divergent promoter cassette was then excised as a Pst $\mathrm{I}-E c o \mathrm{RI}$ fragment and ligated into $\mathrm{pDH} 5$, digested with the same enzymes, to yield pIJ4268. The promoter cassette was further excised as a convenient SpbI fragment for creating pI J4274 and pI J4275 (see Results). [For the record, pIJ6017 carries a variant of $\mathrm{p} t i p A$, $\mathrm{ptip} A^{*}$, with an extra $\mathrm{G}$ inserted 64 bases upstream of the transcriptional start site (Takano, 1993), whereas pAK113 carries the wild-type ptip $A$; in some situations $(H$. Wang, personal communication) ptip $A^{*}$ has been reported to act as a stronger promoter but this was not significant in the present work. In $\mathrm{pIJ} 4274$, ptip $A^{*}$ was responsible for transcription of wbiE-ORFI-VII.]

\section{RESULTS}

\section{Construction of S. coelicolor strains deleted for the whiE gene cluster}

A prerequisite for studies of the biochemical functions of the various whiE genes was a set of strains containing defined deletions in the whiE locus. A primary deletion of the known whiE-ORFI-VII genes was engineered using a marker-replacement ('suicide') vector, pIJ4263 (Fig. 3). On this plasmid, the hygromycin resistance gene (byg) of Streptomyces bygroscopicus (Malpartida et al., 1983) replaced the $S p b \mathrm{I}$ fragment that includes the seven $w b i E$ genes on pI 2156 sequenced by Davis \& Chater (1990), leaving $1.1 \mathrm{~kb}$ of DNA from the whiE locus on either side of hyg. The starting strain for transformation by $\mathrm{pIJ} 4263$ was $S$. coelicolor CH999 (McDaniel et al., 1993a), which carries an engineered replacement of the act gene cluster in the chromosome with the ermE gene $(\Delta a c t)$. It also contains a mutation in the red gene cluster controlling biosynthesis of the red prodigionine pigments. Southern hybridization of transformants of $\mathrm{CH} 999$ by $\mathrm{pIJ} 4263$ demonstrated that they arose by the expected single crossover, either upstream or downstream of whiE-ORFI-VII, in approximately equal numbers. Both classes of recombinants had normal sporulation and grey-pigmented spores. One of the strains was propagated on a medium lacking thiostrepton but containing hygromycin to yield the thiostrepton-sensitive $\triangle a c t \Delta w h i E$ strain YU105. Southern

Table 1. S. coelicolor A3(2) derivatives

\begin{tabular}{|c|c|c|}
\hline Strain & Genotype* & Reference \\
\hline B 60 & bis $A 1$ ura $A 1$ str $A 1$ act-160 (actI-ORFI) SCP2 ${ }^{+}$) & \multirow{2}{*}{ Rudd \& Hopwood (1979) } \\
\hline B78 & bis $A 1$ ura $A 1$ str $A 1$ act-178 (actI-ORF2) $\left.\mathrm{SCP}^{+}\right\}$ & \\
\hline C107 & whiE107 SCP1 ${ }^{+} \mathrm{SCP} 2^{+}$ & \multirow{3}{*}{ Chater (1972) } \\
\hline $\mathrm{C} 124$ & $w b i E 124 \mathrm{SCP}^{+} \mathrm{SCP}^{+}$ & \\
\hline $\mathrm{CH} 1$ & $\operatorname{pro} A 1 \arg A 1 \operatorname{redE} 60$ & \\
\hline $\mathrm{CH} 5$ & $\begin{array}{l}\operatorname{pro} A 1 \arg A 1 \text { redE60 actI-ORF3/act VII } \\
\text { uncoupled }\end{array}$ & \multirow{4}{*}{ Khosla et al. (1992) } \\
\hline $\mathrm{CH} 6$ & $\operatorname{pro} A 1 \arg A 1$ redE 60 actI-ORF3 (mis-sense) & \\
\hline $\mathrm{CH} 12$ & $\operatorname{pro} A 1 \arg A 1 \operatorname{redE} 60 \operatorname{actI-ORF3}$ (frame-shift) & \\
\hline $\mathrm{CH} 13$ & $\operatorname{pro} A 1 \arg A 1 \operatorname{redE} 60$ actI-ORF3 (deletion) & \\
\hline CH999 & $\operatorname{pro} A 1 \arg A 1$ redE60 $\triangle a c t(e r m E)$ & McDaniel et al. (1993a) \\
\hline YU105 & pro $A 1 \arg A 1$ redE60 $\Delta a c t(e r m E) \Delta w b i E(b y g)$ & \multirow{7}{*}{ This work } \\
\hline YU106 & $\operatorname{pro} A 1 \arg A 1 \operatorname{redE} 60 \Delta w b i E$ (byg) & \\
\hline YU107 & $\operatorname{pro} A 1 \arg A 1 \operatorname{redE} 60 \Delta a c t(\mathrm{ermE})$ wbiE & \\
\hline YU108 & $\operatorname{pro} A 1 \arg A 1 \mathrm{redE} 60 \Delta a c t(\mathrm{erm} E)$ whiE & \\
\hline YU114 & $\operatorname{pro} A 1 \arg A 1$ redE60 $\Delta a c t(e r m E)$ wbiE & \\
\hline YU116 & $\operatorname{pro} A 1 \arg A 1$ redE60 $\Delta a c t(e r m E)$ whiE & \\
\hline YU127 & $\operatorname{pro} A 1 \arg A 1$ redE60 actI-ORF3 (deletion) wbiE & \\
\hline
\end{tabular}

*All strains are $\mathrm{SCP} 1^{-} \mathrm{SCP} 2^{-}$except where indicated. 
Table 2. Plasmids

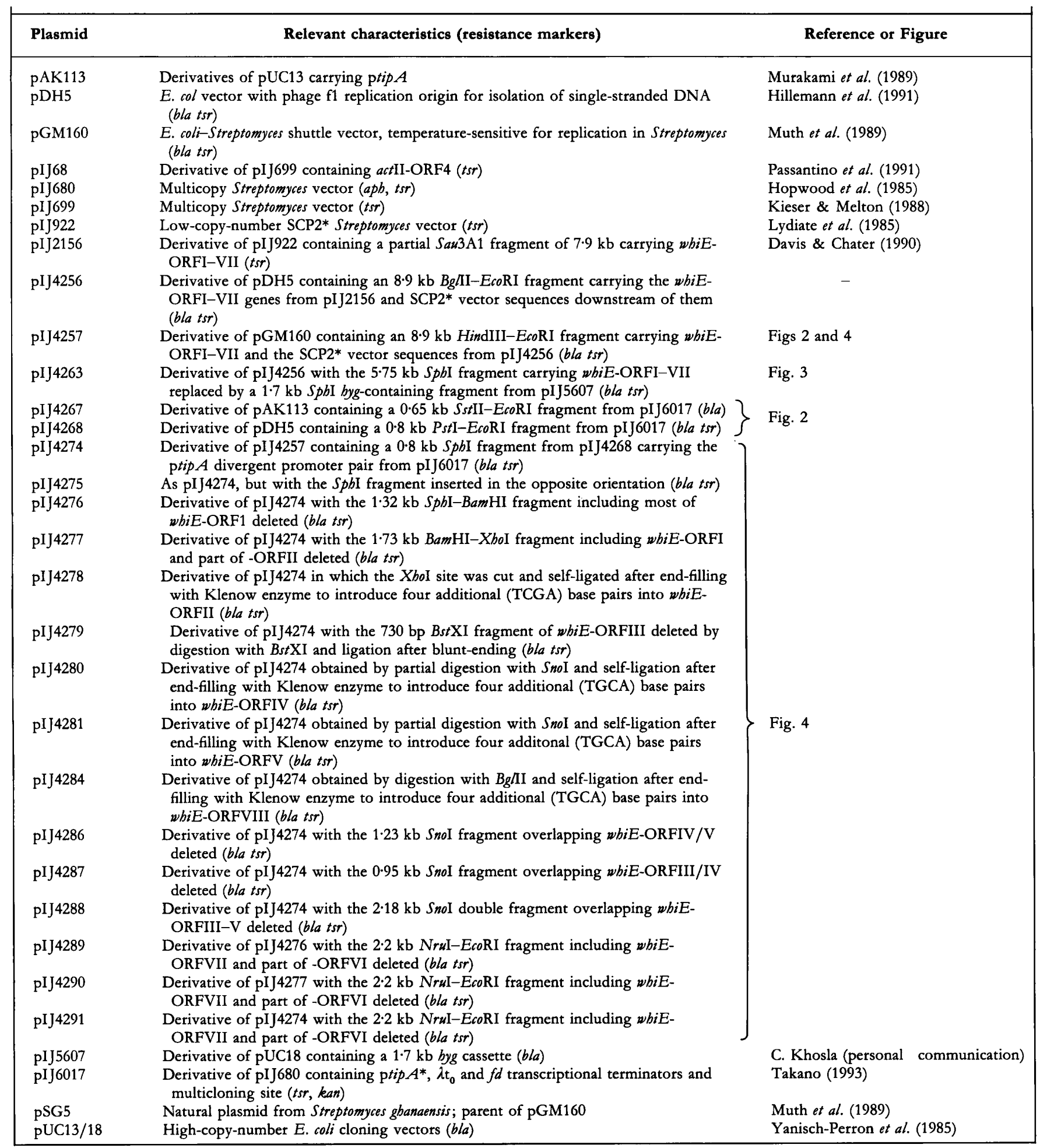

hybridization confirmed that this strain arose by the expected second crossover, which replaced whiEORFI-VII on the chromosome by byg. YU105 sporulated as well as the $w h i E^{+} \mathrm{CH} 999$ but lacked spore pigmentation; like CH999, YU105 produced no actinorhodin or any pigmented precursor of it.
In order to construct an $a c t^{+} \Delta w b i E$ recombinant, a protoplast fusion was made between $\mathrm{YU} 105$ and $\mathrm{CH} 1$, the act $^{+}$progenitor of CH999 (Khosla et al., 1992). After protoplast regeneration, followed by selection for hygromycin-resistant colonies (thereby ensuring inheritance of the whiE deletion), they were screened for blue 


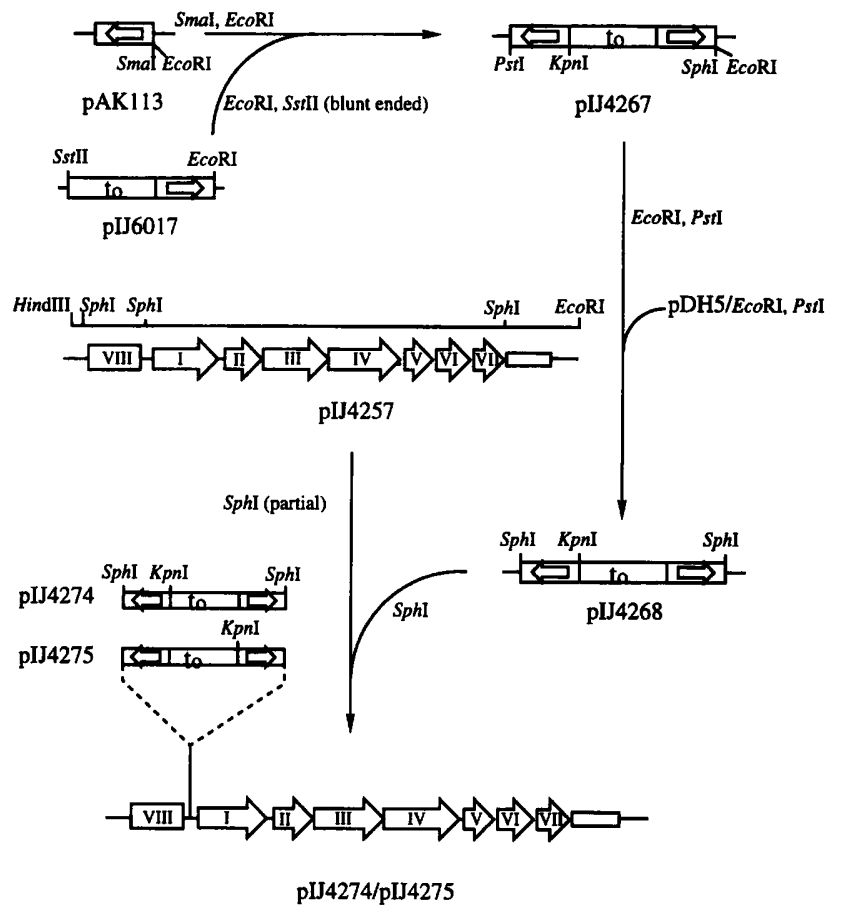

Fig. 2. Construction of the bidirectional ptipA divergent promoter cassette (small arrows in rectangles) and its cloning into the whiE cluster. Only selected restriction sites are shown. The Sphl sites on plJ4268 are from the polylinker flanking the promoter cassette; the asymmetric Kpnl site served to determine its orientation in plJ4274 and plJ4275.

pigment (actinorhodin) production. The resulting colonies were checked for lincomycin sensitivity by replica plating (the ermE marker that replaces the act cluster in CH999 and YU105 confers lincomycin resistance). This yielded the $a c t^{+} \Delta w b i E$ strain YU106. Southern hybridization confirmed that YU106 had the expected genomic arrangement at the whiE and act loci.

To confirm that neither YU105 nor YU106 had suffered any mutation, apart from deletion of whiE-ORFI-VII, that might have had potential to affect spore pigmentation, pIJ4257 (a ColE1-pSG5 bifunctional replicon carrying the whiE gene cluster fragment from $\mathrm{pIJ2156) \text {was }}$ introduced into the chromosome of both strains by recombination, whereupon normal grey-pigmented spores were produced. (These strains were readily obtained after a period of growth at $39^{\circ} \mathrm{C}$, making use of the temperature-sensitive replication properties of the pSG5 replicon.)

\section{Induced expression of the whiE genes}

Expression of the whiE genes on plasmids. The thiostrepton-inducible $\mathrm{ptip} A$ was used to express the $w b i E$ genes ectopically. The wbiE gene cluster includes a gene (ORFVIII) transcribed divergently from the whiEORFI-VII operon (Fig. 1). Therefore two copies of $\mathrm{ptip} A$ were engineered back-to-back as a cassette bounded by convenient restriction sites (see Methods). pI J4274 and
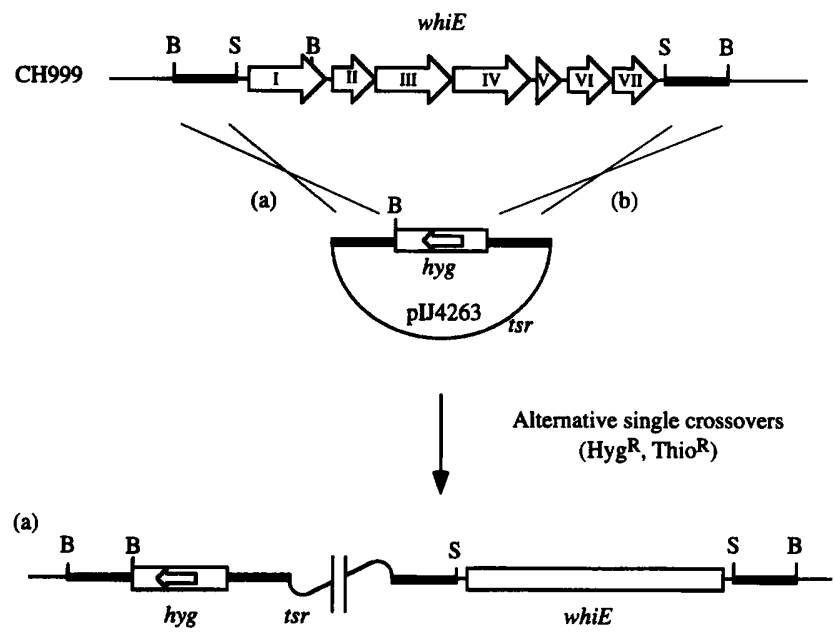

(b)

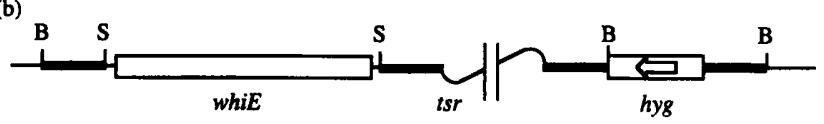

Second crossover

(HygR, Thios)

YU105

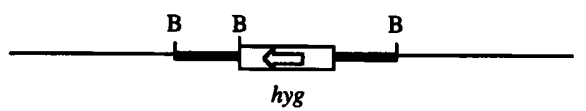

Fig. 3. Strategy for replacement of the whiE gene cluster with the hyg gene. At the top is shown a restriction map of the whiE region of the S. coelicolor CH999 chromosome and, aligned with it, the suicide vector plJ4263 containing the hyg gene replacing seven whiE ORFs and with $\sim 1.1 \mathrm{~kb}$ of additional DNA on each side (black boxes). The vector, derived from pDH5, is equipped with a phage $f 1$ origin of replication so that singlestranded DNA can readily be generated in $E$. coli for efficient transformation of Streptomyces strains (Hillemann et al., 1991; Yu et al., 1994), even when they contain restriction systems. After a first crossover in region (a) or (b), colonies resistant to both thiostrepton (conferred by tsr) and hygromycin (conferred by hyg) were selected. YU105, which was recovered by passaging these doubly resistant strains on a medium lacking thiostrepton, was the result of double crossing-over. B, BamHI restriction site; S, Sphl restriction site.

pIJ4275 were made by inserting the ptip $A$ cassette, in opposite orientations, into the SphI site upstream of the whiE-ORFI-VII operon on pI J4257 (Fig. 2). (For expression of the whiE genes on these plasmids, only one member of the pair of tip $A$ promoters is relevant, since the divergent whiE-ORFVIII is truncated, but both members of the pair of promoters were needed for later experiments: see below under Induced expression of whiE genes in the chromosome.)

The mycelium of young transformants of YU105 ( $\Delta a c t$ $\Delta w b i E$ ) by both plasmids was yellow-green in the presence of thiostrepton; a small amount of yellow-green pigment appeared in the medium, but only after prolonged incubation ( $\sim 1$ week). The mycelium and medium were colourless in the absence of thiostrepton, proving that 


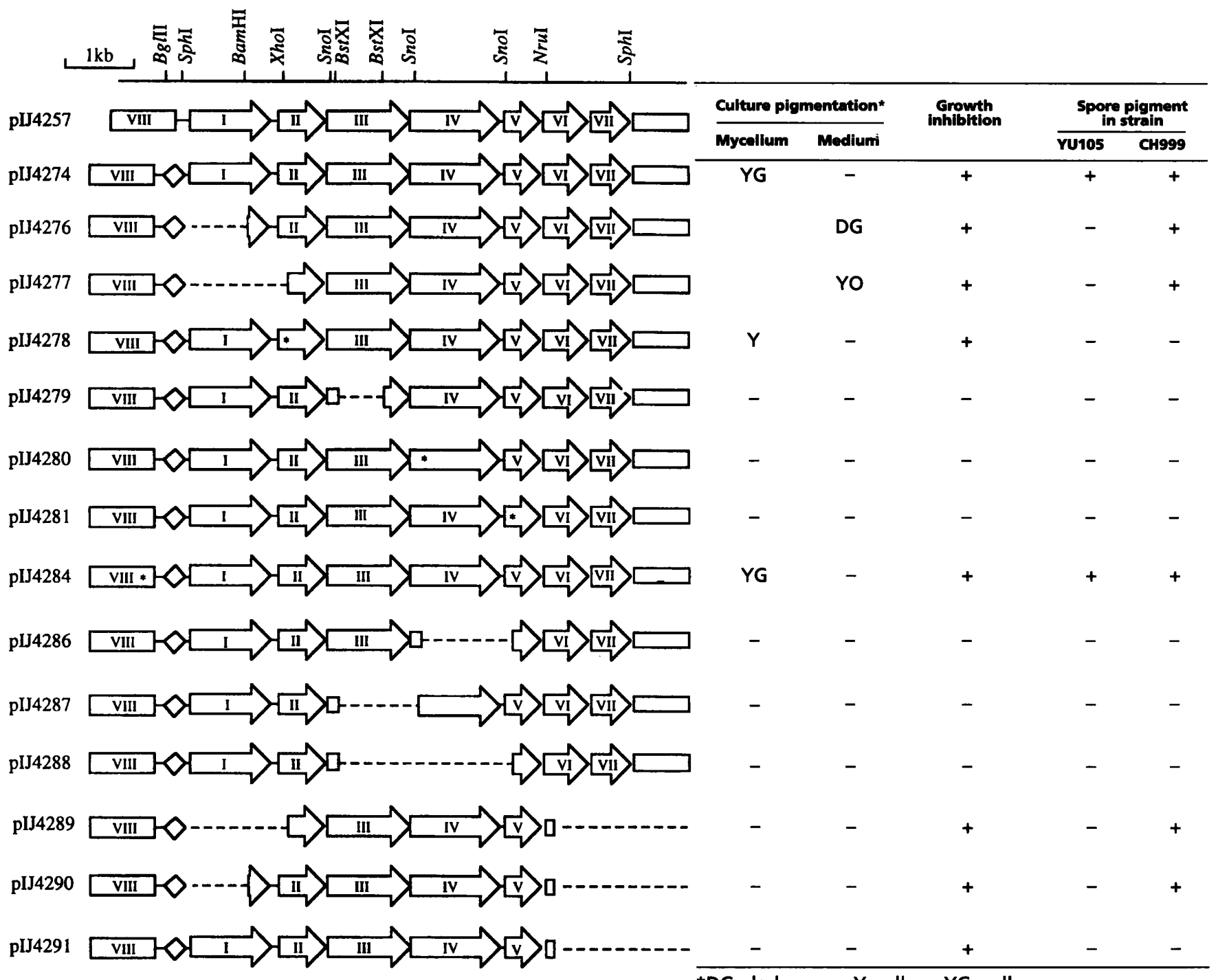

*DG, dark green; $Y$, yellow; YG, yellow-green; YO, yellow-orange.

Fig. 4. Organization of ORFs in whiE expression clones and phenotypes conferred by each plasmid on host strains. The restriction sites used for clone construction are indicated for plJ4257, shown at the top. The rectangle to the left is the $\mathrm{N}$ terminal part of whiE-ORFVIII and the one to the right is the $1.1 \mathrm{~kb}$ unsequenced DNA segment located downstream of whiE-ORFVII in the original whiE clone plJ2156 (Davis \& Chater, 1990). The symbol $\diamond$ indicates the divergent ptipA promoter pair cassette, and the asterisk in certain ORFs indicates the presence of a frame-shift mutation.

pigment production depended on expression of whiEORFI-VII. Unexpectedly, spores harvested from the yellow-green cultures grown in the presence of thiostrepton failed to grow on new plates containing the antibiotic. The cultures could be maintained either by successive propagation of mycelium from thiostreptoncontaining plates, or by plating spores without thiostrepton and transferring the resulting mycelium to thiostrepton-containing medium. The inhibition by thiostrepton affected spore germination or very early growth of the cultures because, although complete inhibition occurred when thiostrepton was added up to $24 \mathrm{~h}$ after spore plating, the antibiotic did not affect growth after colonies were visible. Attempts to grow the strains in liquid medium (TSB) with thiostrepton resulted in granular mycelial clumps in the first 3-4d. Later, the clumps mostly lysed and the broth became deeper yellowgreen. The significance of these results is discussed below.

In order to explore the functions of each whiE ORF, a series of deletions and frame-shift mutations were made on pIJ4274, as described in Table 2 and Fig. 4. Each plasmid was introduced into both YU105 ( $\triangle a c t \Delta w b i E)$ and $\mathrm{CH} 999$ ( $\Delta a c t w h i E^{+}$) and the effects on spore pigmentation, colony pigmentation and the growth inhibition described above were examined in the presence of thiostrepton. The results are summarized in Fig. 4.

The spore pigmentation phenotypes of YU105 derivatives carrying each of the plasmids showed that each of whiEORFI-V, and at least one of ORFVI and VII, are 
necessary for spore pigmentation, since this was abolished by all of the lesions in these ORFs (separate deletions of ORFVI and VII were not made). Unexpectedly, eight plasmids (pI J4278-4281, pI J4286-4288 and pIJ4291) even caused $S$. coelicolor $\mathrm{CH} 999$ to lose its normal spore pigmentation. This could be reversed by isolating strains in which the whiE-containing fragment had become integrated at single copy number by recombination into the relevant region of the whiE locus in the chromosome, again making use of the temperature-sensitive replication properties of the PSG5 replicon. Two features are shared by the eight clones that caused loss of spore pigmentation in CH999: a plasmid-borne whiE promoter for ORFI-VII, which was not inactivated by insertion of the $\mathrm{ptip} A$ cassette because the $S p h \mathrm{I}$ site is about $100 \mathrm{bp}$ upstream of the transcription start site (Brian, 1992), and a lesion in at least one of these seven ORFs on the plasmid. We speculate that a specific factor, such as an activator of the $w h i E$ promoter, is involved in regulation of spore pigment production. Introduction into $\mathrm{CH} 999$ of multiple copies (about 50) of the whiE promoter on these plasmids would, on this hypothesis, cause the endogenous activator to be sequestered on mutant copies of the plasmid-borne whiE operon, so that the chromosomal whiE promoter would not be activated.

Mutations involving any of $w h i E-O R F s$ III-VII abolished production of the yellow-green colony pigment induced by the complete set of ORFs I-VII on pIJ4274/5 (Fig. 4). In contrast, inactivation of ORFI, ORFII or both (pI J4276, pI J4278 or pI J4277) modified the pigmentation phenotype rather than abolishing it. Inactivation of ORFI alone dramatically increased pigmentation in the medium, giving rise to a dark green colour. This suggested that the ORFI product may play a role in retention of spore pigment (or a precursor) at some appropriate intracellular or cell-surface site. In contrast, inactivation of ORFII alone changed the colour of pigmentation from a greenish to a yellowish hue. Thus ORFII is likely to control a step in spore pigment biosynthesis. Again, deletion of ORFI in the presence of the ORFII mutation (pIJ4277 compared with pIJ4278) increased extracellular pigmentation: YU105/pI J4278 made a small amount of yellow pigment in the substrate mycelium, whereas YU105/pIJ4277 produced copious yellow-orange pigmentation in the medium.

The finding of a dark-green pigment in the medium of YU105/pI J4276 (lacking whiE-ORFI) echoes the findings of Horinouchi \& Beppu (1985), who reported production of a diffusible 'brown' pigment when expression of what is now known to be an incomplete set of whiE genes on their pARC1 plasmid (Davis \& Chater, 1990) was activated in Streptomyces lividans; pARC1 would have lacked $w h i E-O R F I$.

Certain of the expression clones carrying whiE deletions displayed the growth inhibition previously associated with the presence of the complete set of whiE ORFs I-VII on $\mathrm{pIJ} 4274 / 5$. The inhibition clearly involved ectopic expression of the whiE genes induced by thiostrepton, rather than activation of the tip $A$ promoter itself or a direct effect of thiostrepton, because other clones carrying the same promoter and challenged with an equal amount of thiostrepton did not show the same effect. A clue to the cause of the inhibition was given by its complete correlation with the presence on the plasmid of a subset of three of the wbiE genes, ORFIII-V (Fig. 4). Studies of the expression in $\mathrm{CH} 999$ of sets of genes encoding the PKSs for the various aromatic antibiotics (Fig. 1) (McDaniel et al., 1993a, 1994) have established that a 'minimal' PKS consisting of just three subunits, a ketosynthase (KS), chain length factor (CLF) and acyl carrier protein (ACP), is competent to synthesize the basic carbon backbone of the polyketide product. wbiE-ORFIII, IV and V are the homologues of the genes encoding these three subunits. Thus the inhibition of early growth is associated with the potential to make a polyketide backbone. Since biosynthesis of the carbon chains of aromatic polyketides is biochemically related to that of fatty acids, induction of high levels of the whiE minimal PKS at an inappropriate stage during growth might lead to exhaustion of precursors, auxiliary enzymes and/or cofactors required for fatty acid metabolism. Alternatively, the metabolic products of the whiE-PKS might be toxic to the host or be processed into novel fatty acids that could disturb cell physiology.

Induced expression of the whiE genes in the chromosome. To study the effects of induced expression of the $w b i E$ ORFs in their normal chromosomal location, some of the DNA fragments used for induced expression of the whiE ORFs on plasmids (Fig. 4) were inserted into the chromosome at the whiE locus. To do so, derivatives of YU105 ( $\triangle a c t \Delta w b i E)$ containing each plasmid were grown at $39^{\circ} \mathrm{C}$ with selection for thiostrepton resistance (encoded by the $t s r$ gene on the vector), and the resulting colonies were propagated on a medium lacking both thiostrepton and hygromycin (byg replaces whiEORFI-VII in YU105) to recover the appropriate $\mathrm{Tsr}^{\mathrm{S}} \mathrm{Hyg}^{\mathrm{s}}$ double crossover product. Southern hybridization confirmed that the byg-containing fragment of YU105 had indeed been replaced in these strains (YU107, YU108, YU114, YU116) by the whiE genes from the relevant plasmid. pGM160 was then introduced into each strain as an autonomous plasmid to render it thiostrepton resistant so that the pair of tip $A$ promoters inserted into the chromosomes of the recombinant strains could be induced. The phenotypes of the recombinants in respect of colony and spore pigmentation were then observed (Table 3).

In the absence of thiostrepton, YU107 had the same phenotype as $\mathrm{CH} 999\left(\Delta a c t w h i E^{+}\right)$: an absence of pigment in the substrate mycelium and medium and the normal grey spore colour. This confirmed that the naturally developmentally regulated $w h i E$ promoters were not affected by insertion of the ptip $A$ cassette. In the presence of thiostrepton, a yellow-green mycelial pigment, indistinguishable from that induced in YU105/pI J4274, was seen. For YU108, lacking whiE-ORFI, induction of whiE expression by addition of thiostrepton caused abundant dark green pigment to appear in the medium of young cultures; this behaviour was the same as for 
Table 3. Phenotypes of $\Delta a c t$ strains with sets of chromosomal whiE genes expressed by ptipA induction

\begin{tabular}{|c|c|c|c|c|c|}
\hline \multirow[t]{2}{*}{ Strain } & \multirow{2}{*}{$\begin{array}{l}\text { Corresponding } \\
\text { plasmid* }\end{array}$} & \multirow{2}{*}{$\begin{array}{c}\text { whiE } \\
\text { ORFs } \\
\text { present }\end{array}$} & \multicolumn{2}{|c|}{ Culture pigmentation } & \multirow{2}{*}{$\begin{array}{c}\text { Spore } \\
\text { pigmentation }\end{array}$} \\
\hline & & & Mycelium & Medium & \\
\hline YU107 & pIJ4274 & I-VIII & Yellow-green & - & Grey (wild-type) \\
\hline YU108 & pIJ4276 & II-VIII & & Dark green & Colourless \\
\hline YU116 & pIJ4277 & III-VIII & & Light yellow & Colourless \\
\hline YU114 & pIJ4284 & I-VII & Yellow-green & - & Greenish \\
\hline
\end{tabular}

*See Fig. 4.

Table 4. Complementation of three different actl-ORF3 (ACP) mutants by whiE-ORFV

\begin{tabular}{|c|c|c|c|c|}
\hline \multirow[t]{2}{*}{$\begin{array}{l}\text { Plasmids used for } \\
\text { complementation }\end{array}$} & \multirow[t]{2}{*}{$\begin{array}{l}\text { CH999 (Aact } \\
\text { control) }\end{array}$} & \multicolumn{3}{|c|}{$\begin{array}{l}\text { Culture pigmentation induced in actI-ORF3 } \\
\text { (ACP) mutants }\end{array}$} \\
\hline & & $\begin{array}{c}\mathrm{CH} 6 \\
\text { (mis-sense) }\end{array}$ & $\begin{array}{c}\text { CH12 } \\
\text { (frame-shift) }\end{array}$ & $\begin{array}{c}\text { CH13 } \\
\text { (deletion) }\end{array}$ \\
\hline $\begin{array}{l}\text { pIJ4277 } \\
(w h i E-O R F I I I-V I I)\end{array}$ & Yellow-orange & Brown & Purple & Blue \\
\hline $\begin{array}{l}\text { pIJ } 4287 \\
\text { (whiE-ORFI, II, V, VI, VII) }\end{array}$ & Colourless & Brown & Purple & Blue-purple \\
\hline
\end{tabular}

YU105/pI J4276. In comparable fashion, YU116, lacking both whiE-ORFI and -ORFII, secreted a light-yellow pigment resembling that made by YU105/4277 into thiostrepton-containing medium from the early stages of growth. The spores of YU108 and YU116 were unpigmented. These results confirmed the conclusions, from YU105/pIJ4276 and YU105/pIJ4277 respectively, that whiE-ORFI is required for spore pigmentation, and when expressed ectopically in the vegetative mycelium along with the products of $w h i E-O R F I I-V I I$ it leads to retention of the resulting spore pigment-related metabolites in or on the mycelium. A role for whiE-ORFVIII in normal spore pigmentation was demonstrated for the first time by the spore colour of the ORFVIII mutant YU114. This was greenish, compared with the normal grey colour of whi $E^{+}$strains.

\section{Co-operation between components of the act and whiE minimal PKS subunits induced by ectopic expression}

Complementation of act-PKS mutations by the corresponding whiE-PKS genes. Attempts were made to complement mutations in each of the three act minimal PKS genes by the corresponding whiE genes. The first experiments involved lesions in actI-ORF3, encoding the act-ACP. Since none of the 12 'classical' actI mutants of Rudd \& Hopwood (1979) mapped in ORF3 (Fernández-Moreno $e t$ al., 1992; Sherman et al., 1992), three constructed null mutants (CH6, CH12 and CH13) (Khosla et al., 1992) were used. The three strains were transformed with two of the expression plasmids (Fig. 4) carrying the ACPencoding whiE-ORFV, either as the only member of the minimal whiE-PKS gene set (pIJ4287) or as part of the trio of such genes that includes the KS and CLF genes (pIJ4277). The results (Table 4) clearly demonstrated formation of a functional 'hybrid' PKS in each of the six combinations, since all strains produced diffusible pigment in the presence but not in the absence of thiostrepton.

Interestingly, the amount of pigment induced by both plasmids in $\mathrm{CH} 6$ was considerably less than in $\mathrm{CH} 12$ and $\mathrm{CH} 13$. A possible explanation lies in the difference between the ACP defects in the three actI-ORF3 mutants. CH6 carries a mis-sense mutation that changes serine- 42 (the site of attachment of the 4'-phosphopantetheine prosthetic group) of the ACP to alanine; $\mathrm{CH} 12$ contains a frame-shift mutation near the $5^{\prime}$ end of the ACP gene; in $\mathrm{CH} 13$ a precise deletion has removed the whole of the gene. In $\mathrm{CH} 6$ the mutant act-ACP could probably associate with the other two act-PKS subunits to produce an inactive PKS, lacking the acyl carrier function because of a failure to attach the $4^{\prime}$-phosphopantetheine prosthetic group. The functional whiE-ACP component, supplied from the expression of whiE-ORFV on the plasmids, might not compete fully with the non-functional act-ACP to associate with the other two act-PKS components, and 
Table 5. Complementation of actl-ORF1(KS) and actl-ORF2 (CLF) mutants by whiEORFIIIIV

\begin{tabular}{|c|c|c|c|c|c|c|}
\hline \multirow[t]{2}{*}{ act mutants } & \multicolumn{6}{|c|}{$\begin{array}{l}\text { Culture pigmentation induced by plasmids carrying various whiE } \\
\text { PKS genes (encoding the PKS subunits in parentheses) }\end{array}$} \\
\hline & $\begin{array}{c}\text { pIJ4277 } \\
\text { (KS, CLF, } \\
\text { ACP) }\end{array}$ & $\begin{array}{c}\text { pIJ4279 } \\
\text { (CLF, } \\
\text { ACP) }\end{array}$ & $\begin{array}{c}\text { pIJ4280 } \\
(\mathrm{KS}, \mathrm{ACP})\end{array}$ & $\begin{array}{c}\text { pIJ4281 } \\
(\mathrm{KS}, \mathrm{CLF})\end{array}$ & $\begin{array}{l}\text { pIJ4286 } \\
\text { (KS) }\end{array}$ & $\begin{array}{l}\text { pIJ4287 } \\
\text { (ACP) }\end{array}$ \\
\hline $\mathrm{B} 60$ (act-KS mutant) & - & - & Purple & Dark green & Blue & - \\
\hline B78 (act-CLF mutant) & - & Dark green & - & Dark green & - & - \\
\hline
\end{tabular}

-, Same phenotype as host strain without plasmid.

so the proportion of functional 'hybrid' PKS would be small. In contrast, the frame-shift and deletion mutants (CH12 and $\mathrm{CH} 13$ ) would not contain complete act-ACP protein. This hypothesis is also compatible with the different performances of $\mathrm{pIJ} 4277$ and pI J4287. As well as providing a functional whiE-ACP, pIJ4277 contains the genes encoding the whiE-KS and wbiE-CLF, whereas pIJ4287 encodes only the whiE-ACP. pIJ4277 induced much less diffusible pigment than pIJ4287, consistent with the idea that the whiE-ACP encoded by $\mathrm{pIJ} 4277$ was efficiently sequestered by the whiE-KS and wbiE-CLF, making it less available to interact productively with the corresponding act-PKS components.

As a further test of complementation of an act-ACP lesion by $w h i E-O R F V$, this time at single copy number in the chromosome, the wild-type whiE cluster of $\mathrm{CH} 13$ was replaced by the 'mutant' whiE cluster on $\mathrm{pIJ} 4277$ by double crossing-over. To do so, $\mathrm{CH} 13 / \mathrm{pIJ} 4277$ was transferred to $39^{\circ} \mathrm{C}$ and selection was made for a thiostrepton-sensitive recombinant that failed to produce blue pigment and had colourless spores. Southern blotting confirmed that this strain, YU127, had the expected deletion of ORFI and II at the chromosomal whiE locus. One or other of two plasmids carrying tsr was then introduced into YU127: these were pGM160 and pIJ68 (Passantino et al., 1991). pIJ68 is a multi-copy plasmid that carries the activator for the actinorhodin gene cluster, actII-ORF4 (Fernández-Moreno et al., 1991). When the resulting strains were grown in the presence of thiostrepton, blue diffusible pigment was produced, which was especially abundant in YU127/pI J68.

To test for complementation of a lesion in the act-KS, the mutant was B60 (Rudd \& Hopwood, 1979), with a C (frame-shift) deletion in the $5^{\prime}$ region of actI-ORFI, and for the act-CLF it was B78, with a GC (frame-shift) deletion in the $5^{\prime}$ region of actI-ORF2 (Sherman et al., 1992). Both mutants are $\mathrm{red}^{+}$, so they produce the red prodigionine pigments. They were transformed with a set of six plasmids carrying various combinations of whiEORFIII-V (KS, CLF, ACP) under the control of ptip $A$. Marked pigmentation was induced in $\mathrm{B} 60$ by the three plasmids carrying the wbiE-KS gene (pI J4280, pIJ4281 and $\mathrm{PI}$ J286) and in B78 by the two plasmids carrying the whiE-CLF gene (pI J4279 and pI J4281) (Table 5). The lack of any pigmentation induced by $\mathrm{pIJ} 4277$ in $\mathrm{B} 60$ or $\mathrm{B} 78$ was surprising because this plasmid carries both the whiE$\mathrm{KS}$ and -CLF genes and caused yellow-orange pigment to be induced in the Aact strain YU105 (see above); probably such pigmentation was masked by the red-encoded pigments. As in the case of the actI-ORF3 (ACP) results, we can invoke sequestration of homologous PKS subunits to explain the much lower level of pigmentation (if any) in

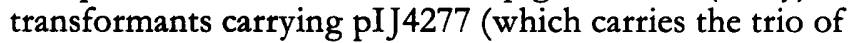
genes for the whiE minimal PKS) than in transformants carrying the plasmids encoding only one or two of the whiE-PKS subunits, which caused much more intense pigmentation.

Complementation of whiE-PKS mutations by the corresponding act-PKS genes. The results described above indicated that each of the components of the whiE minimal PKS can relieve blocks in the actinorhodin biosynthetic pathway caused by lesions in the corresponding act minimal PKS components. To test the possibility of 'reciprocal' complementation, in which act-PKS components could compensate for whiE-PKS lesions to produce spore pigment, use was again made of six of the expression plasmids carrying one, two or all three of the wbiE minimal PKS genes (Fig. 4), which were introduced into YU106 (act $\Delta w b i E)$. pI J4274 was the ' $w b i E^{+}$' control plasmid carrying whiE-ORFI-VII, while YU105 was the $\Delta$ act $\triangle w h i E$ control host. The results are in Table 6.

As expected, the spore pigmentation of both YU105 and YU106 was restored to the normal grey colour by pIJ4274. All six of the wbiE mutant plasmids induced a pale grey spore phenotype in YU106. That this was caused by complementation of the lesions in whiEORFIII-V by the corresponding act minimal PKS genes was shown by the total absence of spore pigmentation induced by these plasmids in the $\Delta a c t$ strain YU105. Moreover, all spore pigmentation induced by the various plasmids in YU106 depended on the presence of thiostrepton in the medium, confirming that it was due to expression of the plasmid-encoded whiE genes. Interestingly, all the plasmids caused some delay in production of blue diffusible pigment (actinorhodin) by YU106, consistent with the possible sequestration of act-PKS subunits by the presence of ectopically expressed whiE minimal PKS components in the substrate mycelium. 
Table 6. Spore pigmentation induced by plasmids carrying sets of whiE genes in mutants lacking whiE-ORFI-VII in the chromosome

\begin{tabular}{|c|c|c|c|}
\hline \multirow[t]{2}{*}{ Plasmid* } & \multirow{2}{*}{$\begin{array}{l}\text { whiE genes on } \\
\text { plasmids }\end{array}$} & \multicolumn{2}{|c|}{ Spore pigment induced in strain $\dagger$} \\
\hline & & $\begin{array}{c}\text { YU105 } \\
\text { (Aact } \Delta w h i E)\end{array}$ & $\begin{array}{c}\text { YU106 } \\
\left(a c t^{+} \Delta w h i E\right)\end{array}$ \\
\hline pIJ4274 & I-VII & + & + \\
\hline pIJ4279 & I, II, IV, V, VI, VII & - & \pm \\
\hline pIJ4280 & I, II, III, V, VI, VII & - & \pm \\
\hline pIJ4281 & I, II, III, IV, VI, VII & - & \pm \\
\hline pIJ4286 & I, II, III, VI, VII & - & \pm \\
\hline pIJ4287 & I, II, V, VI, VII & - & \pm \\
\hline pIJ4288 & I, II, VI, VII & - & \pm \\
\hline
\end{tabular}

*See Fig. 4.

$\dagger+$, Full spore pigmentation (grey); \pm , partial spore pigmentation (pale grey); - , no spore pigmentation (white).

\section{DISCUSSION}

The results reported here have further demonstrated the power of the colony and spore pigmentation phenotypes of $S$. coelicolor for genetic analysis of the act (Rudd \& Hopwood, 1979; Malpartida \& Hopwood, 1984; Sherman et al., 1992; Khosla et al., 1992, 1993), and whiE genes (Hopwood et al., 1970; Chater, 1972; Davis \& Chater, 1990). They have yielded new information about the roles of the various whiE genes in controlling spore pigmentation and about the possibilities for biochemical 'cross-talk' between the act and whiE gene products when the normal regulation of the genes is changed.

\section{Roles of the whiE ORFs}

The whiE DNA sequenced by Davis \& Chater (1990) complemented the two available whiE mutants (C107 and C124: Chater, 1972), but their locations within the whiE cluster are unknown. Therefore the demonstration that at least seven of the eight known wbiE-ORFs (i.e. with the possible exception of either ORFVI or ORFVII) are essential for normal spore pigmentation is novel. Information on the roles of the various ORFs is as follows.

The whiE-ORFI gene product does not resemble known proteins (Davis \& Chater, 1990). Ectopic expression of the gene in the substrate mycelium has provided circumstantial evidence that this protein plays a role in retaining, or targeting, the spore pigment to an appropriate site in or on the spore. This came from the finding that in the absence of whiE-ORFI copious pigmentation appeared in the medium, whereas the pigment remained with the mycelium when whiE-ORFI was present.

The other seven known whiE genes probably all control steps in the biosynthetic pathway for spore pigment. whiE-ORFVIII almost certainly encodes a 'tailoring' enzyme for a late step in the pathway because its disruption resulted in a change in spore colour from grey to greenish.
This echoes an early observation that mutants with greenish spores could readily be isolated after mutagenesis of wild-type $S$. coelicolor and that they mapped to a chromosomal location compatible with that of the later mapped whiE mutations (D. A. Hopwood \& H. M. Kieser, unpublished). Furthermore, the greenish spore pigment phenotype of YU114 (carrying a whiE-ORFVIII mutation) may explain the observation that inserting extra copies of the DNA fragment carrying whiE-ORFI-VII into wild-type $S$. coelicolor, S. lividans or Streptomyces parvulus caused the spore colour to change from grey to greenish (Davis \& Chater, 1990). Perhaps this resulted in a comparative excess of the whiE-ORFVIII substrate, mimicking the effect of blocking the reaction catalysed by whiE-ORFVIII. whiE-ORFVIII resembles $S$. balstedii scbORFC, and both gene products in turn resemble FADlinked hydroxylases (Blanco et al., 1993). Mutation of $s c h-$ ORFC changed the spore colour of $S$. halstedii from green to lilac (Blanco et al., 1993). Perhaps the different spore colours in $S$. coelicolor and $S$. balstedii reflect slight differences in one or more tailoring steps in the biosynthesis of the two spore pigments, since their carbon backbones are probably similar (Yu, 1995). However, the finding that expression of whiE-ORFVIII did not change the mycelial pigmentation (YU114 compared with YU107) might suggest that the tailoring step catalysed by the whiE-ORFVIII product cannot occur on the incompletely elaborated pathway intermediates or shunt products induced by ectopic expression of whiE-ORFII-VII, which are presumed to encode early biosynthetic steps (see below). There may well be further steps in the pathway controlled by unidentified genes acting between the metabolite produced by the $w$ biE-ORFVII product and the substrate for the whiE-ORFVIII product. Alternatively, this might have reflected a lack of expression of the chromosomal copy of whiE-ORFVIII in these experiments.

The roles of $w h i E-O R F I I I-V$ can readily be rationalized because of the resemblance of their protein products to 
the KS, CLF and ACP of other minimal PKSs (Davis \& Chater, 1990; McDaniel et al., 1994), even though their ectopic expression alone gave rise to no obvious pigmentation. Their use to complement mutations in the corresponding genes for the act minimal PKS (see below) confirms their roles.

The whiE-ORFVI gene product resembles the $\mathrm{N}$-terminal half of a family of related gene products in the PKS gene clusters for aromatic polyketide antibiotics. These other genes, many of which show evidence of an ancestral internal duplication to give rise to the C-terminal halves of the gene products (Bibb et al., 1994), are implicated in early cyclization steps of aromatic polyketides (Zhang $e t$ al., 1990; Sherman et al., 1991), probably by catalysing aromatization of the first carbocyclic ring (McDaniel et al., 1994). Similarly, both whiE-ORFII and whiE-ORFVII resemble genes encoding presumed cyclases acting in tetracenomycin biosynthesis after the product of the whiE-ORFVI homologue; these are tcmJ and tcmI respectively (Summers et al., 1992, 1993; McDaniel et al., 1995). Our results strongly support an enzymic role for the whiE-ORFII product in the biosynthetic pathway to spore pigment because of the replacement of a yelloworange shunt product by a dark green compound when ectopic expression of whiE-ORFII was added to that of wbiE-ORFIII-VII.

Taking all these observations together, we predict a likely order of action of the whiE gene products in the biosynthesis of the spore pigment as (III + IV + V) $\rightarrow$ VI $\rightarrow \mathrm{II} \rightarrow \mathrm{VII} \rightarrow \rightarrow \rightarrow$ VIII.

\section{Interactions between act and whiE expression}

Following the discovery that lesions in the act-PKS could be complemented by genes encoding subunits of other aromatic PKSs (Sherman et al., 1992; Khosla et al., 1992, 1993), Kim et al. (1994b) found that an actI-ORFI (KS) null mutant could produce blue pigment (presumably actinorhodin) when the corresponding whiE gene (wbiEORFIII) was introduced into it on a plasmid vector, but that an actI-ORF2 (CLF) mutation was not complemented by its $w h i E$ homologue. In the experiments of Kim et al. (1994b), expression of the introduced whiE-CLF gene relied on readthrough from the $t s r$ vector promoter, which may not have been expressed at a high level, even though this was sufficient for complementation of the act mutation by the corresponding granaticin CLF gene. In our experiments, ectopic expression of the whiE-CLF gene did cause the same actI-CLF mutant to produce pigment, perhaps because of stronger expression of the whiE gene by induction of $\mathrm{p} t i p A$.

We have now shown that deficiencies of any of the three components of the minimal act-PKS - KS, CLF or ACP - can be complemented by the corresponding whiE-PKS subunits to produce diffusible mycelial pigments. These are likely to have been actinorhodin and/or intermediates or shunt products in its biosynthesis, except when the CLF subunit came from the whiE-PKS. This deduction is plausible because exchanging the $\mathrm{KS}$ or $\mathrm{ACP}$ components of various aromatic PKSs has not so far altered the 'programming' of the synthase (McDaniel et al., 1993a, b). In contrast, complementation of an act-CLF mutant by the wbi-CLF subunit may well have given rise to one or more novel metabolites (as is hinted at by the greenish colour of cultures in which the whiE-CLF could have been active: Table 5), because the carbon chain length of the spore pigment is longer than the 16 carbons of actinorhodin (Yu, 1995). Unfortunately, chemical analysis of these cultures did not reveal a clear picture, probably because of the instability of the metabolites (Yu, 1995). The programming capability of the whiE minimal PKS is currently under study in the expression system (McDaniel et al., 1993a) that has proved so successful for other aromatic PKSs (Yu, 1995). Interestingly, the results also provided circumstantial evidence that homologous combinations of minimal PKS subunits may form more effectively than 'hybrid' combinations, implying some degree of protein-protein interaction in the type II aromatic PKS, which was already suggested by the failure of some other KS/CLF hybrids to work effectively (McDaniel et al., 1993a, b; Kim et al., 1994b).

In reciprocal fashion, any of the three act minimal PKS subunits could complement lesions in the corresponding whiE-PKS subunits to produce spore pigment. Again, the possibility remains that this differed from the normal spore pigment when the act-PKS was the source of the CLF, but information is lacking on this point. It was perhaps unexpected that the presence of thiostrepton in the agar medium could lead to efficient enough induction of $\mathrm{ptip} A$ and resulting expression of the act minimal PKS genes to complement lesions in the whiE-PKS genes in an appropriate location, but this turned out not to be a problem.

Clearly, a differential spatial localization of expression of the two sets of PKS genes during normal development of the $S$. coelicolor colony is implied by the findings of Kim $e t$ al. (1994b) and substantiated by our results. This would prevent the potential for biochemical co-operation between the act- and whiE-PKS subunits from being realized; otherwise, mutations in the act- or wbiE-PKS genes would not abolish actinorhodin production or spore pigmentation respectively. The same reasoning implies that the fatty acid synthase(s) (FAS) of the organism also is normally not involved in productive 'cross-talk' with the act-and/or whiE-PKS. Whether this too depends on different spatial and/or temporal expression of the FAS and PKS genes, or whether the FAS gene products are biochemically incapable of interacting productively with those of the act- and/or whiE-PKS genes, is not known, but can now be addressed because putative $S$. coelicolor FAS genes are becoming available (Revill et al., 1995). Already it is clear that one enzyme that may well interact with all minimal FAS and PKS complexes, malonyl CoA : ACP acyltransferase, is likely to be shared by the FAS and at least the act-PKS (Revill et al., 1995). A possible indication of a potential for 'cross-talk' between the FAS and whiE-PKS is the observation of early growth inhibition when all three whiE minimal PKS subunits were ectopically expressed in the substrate mycelium, but it remains to be seen whether this reflects 
an interaction between the synthases to produce abnormal, potentially harmful, fatty acids, or whether the interference is only at the level of competition for substrates or cofactors.

\section{ACKNOWLEDGEMENTS}

We thank Peter Revill for help and many useful discussions during the course of this work. We also thank Peter Revill, Tobias Kieser, Mark Buttner, Keith Chater and Mervyn Bibb for useful comments on the manuscript. The research was supported by grants-in-aid from the BBSRC and the John Innes Foundation.

\section{REFERENCES}

Bibb, M. J., Biro, S., Motamedi, H., Collins, J. F. \& Hutchinson, C. R. (1989). Analysis of the nucleotide sequence of the Streptomyces glaucescens tcmI genes provides key information about the enzymology of polyketide antibiotic biosynthesis. EMBO $J \mathbf{8}$, 2727-2736.

Bibb, M. J., Sherman, D. H., Omura, S. \& Hopwood, D. A. (1994). Cloning, sequencing and deduced functions of a cluster of Streptomyces genes probably encoding biosynthesis of the polyketide antibiotic frenolicin. Gene 142, 31-39.

Blanco, G., Pereda, A., Méndez, C. \& Salas, J. A. (1992). Cloning and disruption of a DNA fragment of Streptomyces balstedii involved in the biosynthesis of a spore pigment. Gene 112, 59-65.

Blanco, G., Brian, P., Pereda, A., Méndez, C., Salas, J. A. \& Chater, K. F. (1993). Hybridization and DNA sequence analyses suggest an early evolutionary divergence of related biosynthetic gene sets for polyketide antibiotics and spore pigments in Streptomyces spp. Gene 130, 107-116.

Brian, P. (1992). $A$ developmentally regulated spore pigment locus from Streptomyces coelicolor $A 3(2)$. $\mathrm{PhD}$ thesis, University of East Anglia, Norwich, UK.

Chater, K. F. (1972). A morphological and genetic mapping study of white colony mutants of Streptomyces coelicolor. J Gen Microbiol 72, 9-28.

Chater, K. F. (1993). Genetics of differentiation in Streptomyces. Annu Rev Microbiol 47, 685-713.

Davis, N. K. \& Chater, K. F. (1990). Spore colour in Streptomyces coelicolor A3(2) involves the developmentally regulated synthesis of a compound biosynthetically related to polyketide antibiotics. Mol Microbiol 4, 1679-1691.

Fernández-Moreno, M. A., Caballero, J. L., Hopwood, D. A. \& Malpartida, F. (1991). The act cluster contains regulatory and antibiotic export genes, direct targets for translational control by the bld $A$ tRNA gene of Streptomyces. Cell 66, 769-780.

Fernández-Moreno, M. A., Martínez, E., Boto, L., Hopwood, D. A. \& Malpartida, F. (1992). Nucleotide sequence and deduced functions of a set of co-transcribed genes of Streptomyces coelicolor A3(2) including the polyketide synthase for the antibiotic actinorhodin. J Biol Chem 267, 19278-19290.

Hillemann, D., Puhler, A. \& Wohlleben, W. (1991). Gene disruption and gene replacement in Streptomyces via single-stranded DNA transformation of integrating vectors. Nucleic Acids Res 19, 727-731.

Hopwood, D. A. \& Khosla, C. (1992). Genes for polyketide secondary metabolic pathways in microorganisms and plants. In Secondary Metabolites: Their Function and Evolution (Ciba Foundation Symposium), pp. 88-112. Chichester: Wiley.
Hopwood, D. A. \& Sherman, D. H. (1990). Molecular genetics of polyketides and its comparison to fatty acid biosynthesis. Annu Rev Genet 24, 37-66.

Hopwood, D. A., Wildermuth, H. \& Palmer, H. M. (1970). Mutants of Streptomyces coelicolor defective in sporulation. J Gen Microbiol 61, 397-408.

Hopwood, D. A., Bibb, M. J., Chater, K. F., Kieser, T., Bruton, C. J., Kieser, H. M., Lydiate, D. J., Smith, C. P., Ward, J. M. \& Schrempf, H. (1985). Genetic Manipulation of Streptomyces: a Laboratory Manual. Norwich: John Innes Foundation.

Horinouchi, S. \& Beppu, T. (1985). Construction and application of a promoter-probe plasmid that allows chromogenic identification in Streptomyces lividans. J Bacteriol 162, 406-412.

Katz, L. \& Donadio, S. (1993). Polyketide synthesis: prospects for hybrid antibiotics. Annu Rev Microbiol 47, 876-912.

Khosla, C., Ebert-Khosla, S. \& Hopwood, D. A. (1992). Targeted gene replacements in a Streptomyces polyketide synthase gene cluster: role for the acyl carrier protein. Mol Microbiol 6, 3237-3249.

Khosla, C., McDaniel, R., Ebert-Khosla, S., Torres, R., Sherman, D. H., Bibb, M. J. \& Hopwood, D. A. (1993). Genetic construction and functional analysis of hybrid polyketide synthases containing heterologous acyl carrier proteins. J Bacteriol 175, 2197-2204.

Kieser, T. \& Melton, R. E. (1988). Plasmid pIJ699, a multi-copy positive selection vector for Streptomyces. Gene 65, 83-91.

Kim, E.-S., Bibb, M. J., Butler, M. J., Hopwood, D. A. \& Sherman, D. H. (1994a). Nucleotide sequence of the oxytetracycline (otc) polyketide synthase genes from Streptomyces rimosus. Gene 176, 1801-1804.

Kim, E-S., Hopwood, D. A. \& Sherman, D. H. (1994b). Analysis of Type II polyketide $\beta$-ketoacyl synthase specificity in Streptomyces coelicolor A3(2) by trans complementation of actinorhodin synthase mutants. J Bacteriol 176, 1801-1804.

Lydiate, D. J., Malpartida, F. \& Hopwood, D. A. (1985). The Streptomyces plasmid SCP2* : its functional analysis and development into useful cloning vectors. Gene 35, 223-235.

McDaniel, R., Ebert-Khosla, S., Hopwood, D. A. \& Khosla, C. (1993a). Engineered biosynthesis of novel polyketides. Science 262, 1546-1550.

McDaniel, R., Ebert-Khosla, S., Hopwood, D. A. \& Khosla, C. (1993b). Engineered biosynthesis of novel polyketides: manipulation and analysis of an aromatic polyketide synthase with unproven catalytic specificities. $J$ Am Cbem Soc 116, 10855-10859.

McDaniel, R., Ebert-Khosla, S., Hopwood, D. A. \& Khosla, C. (1994). Engineered biosynthesis of novel polyketide: actVII and act IV genes encode aromatase and cyclase enzymes, respectively. $J$ Am Chem Soc 116, 10855-10859.

McDaniel, R., Hutchinson, C. R. \& Khosla, C. (1995). Engineered biosynthesis of novel polyketides: analysis of $\mathrm{TcmN}$ function in tetracenomycin biosynthesis. $J \mathrm{Am}$ Chem Soc (in press).

MacNeil, D. J., Gewain, K. M., Ruby, C. L., Dezeny, G., Gibbons, P. H. \& MacNeil, T. (1992). Analysis of Streptomyces avermitilis genes required for avermectin biosynthesis utilizing a novel integration vector. Gene 111, 61-68.

Malpartida, R. \& Hopwood, D. A. (1984). Molecular cloning of the whole biosynthetic pathway of a Streptomyces antibiotic and its expression in a heterologous host. Nature 309, 462-464.

Malpartida, F., Zalacain, M., Jimenez, A. \& Davies, J. (1983). Molecular cloning and expression in Streptomyces lividans of a hygromycin B phosphotransferase gene from Streptomyces bygroscopicus. Biochem Biophys Res Commun 117, 6-12. 
Murakami, T., Holt, T. G. \& Thompson, C. J. (1989). Thiostreptoninduced gene expression in Streptomyces lividans. J Bacteriol 171, 1459-1466.

Muth, G., Nussbaumer, B., Wohlleben, W. \& Puhler, A. (1989). A vector system with temperature-sensitive replication for gene disruption and mutational cloning in streptomycetes. Mol \& Gen Genet 219, 341-348.

O'Hagan, D. (1991). The Polyketide Metabolites. Chichester: Ellis Horwood.

Passantino, R., Puglia, A. M. \& Chater, K. F. (1991). Additional copies of the actII regulatory gene induce actinorhodin production in pleiotropic bld mutants of Streptomyces coelicolor A3(2). J Gen Microbiol 137, 2059-2064.

Revill, W. P., Bibb, M. J. \& Hopwood, D. A. (1995). Purification of a malonyltransferase from Streptomyces coelicolor A3(2) and analysis of its genetic determinant. J Bacteriol 177, 3946-3952.

Rudd, B. A. M. \& Hopwood, D. A. (1979). Genetics of actinorhodin biosynthesis by Streptomyces coelicolor A3(2). J Gen Microbiol 114, 35-43.

Sambrook, J., Fritsch, E. F. \& Maniatis, T. (1989). Molecular Cloning: a Laboratory Manual, 2nd edn. Cold Spring Harbor, NY: Cold Spring Harbor Laboratory.

Shen, B. \& Hutchinson, C. R. (1993). Enzymatic synthesis of a bacterial polyketide from acetyl and malonyl coenzyme A. Science 262, 1535-1540.

Sherman, D. H., Malpartida, F., Bibb, M. J., Kieser, H. M. \& Hopwood, D. A. (1989). Structure and deduced function of the granaticin-producing polyketide synthase gene cluster of Streptomyces violaceoruber TU் 22 . EMBO J 8, 2717-2725.

Sherman, D. H., Bibb, M. J., Simpson, T. J., Johnson, D., Malpartida, F., Fernández-Moreno, M., Martínez, E., Hutchinson, C. R. \& Hopwood, D. A. (1991). Molecular genetic analysis reveals a bifunctional polyketide cyclase/dehydrase gene from Streptomyces coelicolor and Streptomyces violaceoruber, and a cyclase/O-methyltransferase from Streptomyces glaucescens. Tetrabedron 47, 6029-6043.
Sherman, D. H., Kim, E.-S., Bibb, M. J. \& Hopwood, D. A. (1992). Functional replacement of genes for individual polyketide synthase components in Streptomyces coelicolor A3(2) by heterologous genes from a different polyketide pathway. $J$ Bacteriol 174, 6184-6190.

Smith, S. (1994) The animal fatty acid synthase: one gene, one polypeptide, seven enzymes. FASEB J 8, 1248-1259.

Summers, R. G., Wendt-Pienkowski, E., Motamedi, H. \& Hutchinson, C. R. (1992). Nucleotide sequence of the $t c m I I-t c m I V$ region of the tetracenomycin $\mathrm{C}$ biosynthetic gene cluster of Streptomyces glaucescens and evidence that the $t c m N$ gene encodes a multifunctional cyclase-dehydratase- $O$-methyl transferase. $J$ Bacteriol 174, 1810-1820.

Summers, R. G., Wendt-Pienkowski, E., Motamedi, H. \& Hutchinson, C. R. (1993). The tcm VI region of the tetracenomycin $\mathrm{C}$ biosynthetic gene cluster of Streptomyces glaucescens encodes the tetracenomycin F1 monooxygenase, tetracenomycin F2 cyclase, and, most likely, a second cyclase. J Bacteriol 175, 7571-7580.

Takano, E. (1993). $p p G p p$ and antibiotic production in Streptomyces coelicolor $A 3(2) . \mathrm{PhD}$ thesis, University of East Anglia, Norwich, UK.

Yanisch-Perron, C., Vieira, J. \& Messing, J. (1985). Improved M13 vectors and host strains : nucleotide sequences of the M13mp18 and pUC19 vectors. Gene 33, 103-119.

Yu, T.-W. (1995). Physical and functional studies of polyketide syntbase genes of Streptomyces. $\mathrm{PhD}$ thesis, University of East Anglia, Norwich, UK.

Yu, T.-W., Bibb, M. J., Revill, W. P. \& Hopwood, D. A. (1994). Cloning, sequencing and analysis of the griseusin polyketide synthase gene cluster from Streptomyces griseus. J Bacteriol 176, $2627-2634$.

Zhang, H. L., He, X. G., Adefarati, A., Gallucci, J., Cole, S. P., Beale, J. M., Keller, P. J., Chang, C.-J. \& Floss, H. G. (1990). Mutactin, a novel polyketide from Streptomyces coelicolor: structure and biosynthetic relationship to actinorhodin. J Org Chem 55, 1682-1684.

Received 10 March 1995; revised 6 July 1995; accepted 12 July 1995. 